\title{
SENSITIVITY OF MRQAP TESTS TO COLLINEARITY AND AUTOCORRELATION CONDITIONS
}

\author{
DAVID DEKKER
}

RADBOUD UNIVERSITY NIJMEGEN

DAVID KRACKHARDT

CARNEGIE MELLON UNIVERSITY

\author{
TOM A.B. SNIJDERS \\ UNIVERSITY OF GRONINGEN AND UNIVERSITY OF OXFORD
}

\begin{abstract}
Multiple regression quadratic assignment procedures (MRQAP) tests are permutation tests for multiple linear regression model coefficients for data organized in square matrices of relatedness among $n$ objects. Such a data structure is typical in social network studies, where variables indicate some type of relation between a given set of actors. We present a new permutation method (called "double semipartialing", or DSP) that complements the family of extant approaches to MRQAP tests. We assess the statistical bias (type I error rate) and statistical power of the set of five methods, including DSP, across a variety of conditions of network autocorrelation, of spuriousness (size of confounder effect), and of skewness in the data. These conditions are explored across three assumed data distributions: normal, gamma, and negative binomial. We find that the Freedman-Lane method and the DSP method are the most robust against a wide array of these conditions. We also find that all five methods perform better if the test statistic is pivotal. Finally, we find limitations of usefulness for MRQAP tests: All tests degrade under simultaneous conditions of extreme skewness and high spuriousness for gamma and negative binomial distributions.
\end{abstract}

Key words: MRQAP, Mantel tests, permutation tests, social networks, network autocorrelation, collinearity, dyadic data.

\section{Introduction}

The problem of statistical analysis of social network data is an old one (Proctor, 1969). Such data are dyadic in the sense that for a basic set of objects $O_{1}, \ldots, O_{n}$, the observed variables refer to pairs of objects and therefore are doubly indexed, e.g., the variable $Y_{i j}$ refers to the way in which object $O_{i}$ is related to object $O_{j}$. Depending on the application $Y_{i j}$ can be continuous (e.g., trade between nations) or discrete (e.g., binary in trust or no trust between individuals, or count as in frequency of linking between web pages). It has long been understood that the lack of independence of dyadic observations creates a severe barrier to reasonable interpretations of statistical tests (Laumann \& Pappi, 1976; Laumann, Marsden, \& Galaskiewicz, 1977). Indeed, using standard OLS models on dyadic data with a moderate amount of structural autocorrelation (lack of independence among observations within the rows and within the columns of network data) biases the estimates of the second moments to such an extent that it is not uncommon for type I errors of $t$-statistics to exceed 50\% (Krackhardt, 1988), rendering the significance tests unusable for all practical purposes.

Special thanks go to Cajo Ter Braak, Philip Hans Franses, Patrick Houweling, Pierre Legendre, three anonymous reviewers, the associate editor, and the editor for comments.

Requests for reprints should be sent to David Dekker, Econometric Institute, Erasmus University Rotterdam, P.O. Box 1738, 3000DR Rotterdam, The Netherlands. E-mail: ddekker@ few.eur.nl 
Within the past 20 years, two substantially different approaches to solving this problem have been proffered: linear models tested with the aid of the Mantel test (Mantel, 1967), which is often referred to as the quadratic assignment procedure (QAP) in social network studies, and exponential random graph models. The QAP approach (Mantel, 1967; Hubert, 1987; Krackhardt, 1987) provides a specific type of permutation test which keeps intact the dyadic data structure under the permutations. The principle of this test can be applied to many kinds of models, but it is usually applied to linear models for data treated as continuous. Through a series of Monte Carlo simulations, Krackhardt (1988) showed that parameters in an OLS model of network autocorrelated data could be tested using a multiple regression extension of the QAP test, called MRQAP. Exponential random graph models (Holland \& Leinhardt, 1981; Frank \& Strauss, 1986; Wasserman \& Pattison, 1996; Snijders, Pattison, Robins, \& Handcock, 2006), on the other hand, are a family of models for dichotomous or other discrete network data where the focus is on modeling the specific network-related dependence structure. The present paper is in the line with the first approach.

Krackhardt's MRQAP approach had an appeal of simplicity and accessibility and, at the time, it was said that it "... takes an exciting step forward in the analysis of network relations" (Pattison, 1988). It has been frequently used to test models in network research (e.g., Borgatti \& Cross, 2003; Gibbons \& Olk, 2003; Sorenson \& Stuart, 2001; Mizruchi, 1990). However, the simulations used in Krackhardt (1988) to justify this approach were confined to a very restricted set of conditions, namely, that there is no correlation among the independent variables nor a correlation in the population between the dependent variable and any of the independent variables (i.e., the null hypothesis was independence among all variables). More recently, literature on permutation tests has shown that collinearity ${ }^{1}$ might cause problems (e.g., Anderson \& Robinson, 2001). However, in this literature the problem of the typical kind of dependence of matrix data is usually not addressed and no specific attention is given to QAP-like tests (for an exception, see Legendre, 2000).

In this paper we study what the literature on permutation tests in linear models has to offer for MRQAP tests for social network data. We assess the problems that might occur due to collinearity in MRQAP-like tests and whether the different proposed permutation tests are robust against sender and receiver dependence as encountered in social network research. Based on prior results in the literature on permutation tests in linear models, we conjecture about the performance of different MRQAP approaches under various collinearity, skewness, and autocorrelation conditions. Furthermore, we develop a new approach to the MRQAP test that addresses some of the shortcomings of previously used versions of MRQAP. Finally, we evaluate these conjectures through an extensive simulation study with parameter values under the null as well as alternative hypotheses, which are typical for data sets encountered in practice in social network analysis for numerical relational variables. We show how various reasonable permutation methods, all consistent with the spirit of the originally proposed MRQAP tests (including our new method), vary considerably in their ability to provide statistical tests with correct type I error rates and good power properties. The conclusion is that for data that do not have a strongly skewed or granular discrete distribution, and for which a linear model is appropriate, some-but not all—of the MRQAP approaches can perform very well in practice.

\footnotetext{
${ }^{1}$ Here we use the term collinearity to indicate any type of correlation among the independent variables used in an analysis.
} 


\section{QAP Permutation Test for Regression Coefficients}

\subsection{Background}

The QAP approach to studying dyadic data in general has been widely used by researchers in a broad array of disciplines, such as statistics (Oden \& Sokal, 1992), biology (Legendre, 2000), and psychology (Hubert, 1987). The approach was first suggested by the statistician Mantel (1967) to address the epidemiological question of whether the distribution of diseases is geographically clustered. It was Hubert (1987) who adopted the term "quadratic assignment" and who found a vast array of applications for this type of test.

Since these early days, many statisticians have uncovered a variety of techniques for performing these permutation tests and concomitant advantages and disadvantages for different data situations (see Legendre, 2000, for a good review on distance data) (see Anderson \& Legendre, 1999; Anderson \& Robinson, 2001, for good studies on permutation tests in ordinary regression). However, the vast majority of these studies have focused on the type of autocorrelation proposed in the original Mantel study - that is, observations are thought to be autocorrelated to the extent that they are geographically proximal. In Legendre, Lapointe, and Casgrain (1994), autocorrelation was phylogenetic and not geographic.

The focus of this paper, by contrast, is network data that are commonly considered to have a different kind of autocorrelation structure. We consider types of autocorrelation, common in dyadic data, where observations are autocorrelated if they are in the same row (emanate from the same sender) or in the same column (go to the same receiver) (e.g., Holland \& Leinhardt, 1981). We wish to assess whether these insights into the properties of the QAP permutation tests hold up under conditions of network autocorrelated data.

\subsection{Bivariate $Q A P$}

The MRQAP test was developed as an extension of the bivariate QAP model. Mantel (1967) first proposed the quadratic assignment procedure (QAP) to assess association between spatial and temporal distance data using a regression approach. These data can be represented in square $n \times n$ matrices where the elements reflect Euclidean distances between numerical attributes of $n$ objects $\left(O_{j}\right.$, where $\left.j=1, \ldots, n\right)$ and the diagonal is structurally zero. Application of the QAP method was subsequently proposed for other structurally autocorrelated data such as social network data where square matrices reflect relations between individuals (e.g., Baker \& Hubert, 1981; Krackhardt, 1987). Matrices that reflect social networks are specific in that dyadic relations are often asymmetric. This is usually not the case with the distance matrices analyzed in other fields using the Mantel test, although it happens from time to time.

Assume we wish to use QAP to assess statistical significance of some measure of association $\Gamma_{Y X}$ between distance or network variables $Y$ and $X$, where both $Y$ and $X$ are $n \times n$ matrices referring to a set of $n$ objects $O_{1}, \ldots, O_{n}$, and where the $(i, j)$ elements of the matrices refer to aspects of how objects $i$ and $j$ are related. The objects are points in space for distance data, and network vertices (e.g., social actors) for network data. The data matrices could be symmetric or nonsymmetric, depending on the situation; this distinction is of no concern to the discussion given here. The association coefficient $\Gamma_{Y X}$ could be, e.g., the elementwise Pearson product moment correlation between $X$ and $Y$ (to calculate association measures variables are reshaped into vectors). For example, Kadushin (1995) shows how French financial elite friendship ties are correlated with (dis)similarity on several attribute variables, such as political preference, educational institute, and club membership.

In the QAP we randomly permute the $n$ objects $O_{j}$ to obtain a new order of objects $O_{\pi(j)}$, the permutation being denoted by $\pi$. For variable $Y$ we calculate the distances or network variables between all objects in the order $O_{\pi(j)}$; the matrix obtained is called $\pi(Y)$. This is equivalent 
to applying the same permutation to the row and column indices of $Y$. Since the permuted structure is isomorphic to the original, all the structural features of the original matrix are retained, except for those referring to the order of the objects. The result is that we generate a random data set $\pi(Y)$ with the same row-column interdependence of observations as there is in $Y$.

Under the null hypothesis of no association between $Y$ and $X$, the observed association $\Gamma_{\pi(Y) X}$ between $\pi(Y)$ and $X$ is a random association that was drawn from the same underlying distribution as the actually observed association $\Gamma_{Y X}$. Now we can simulate this distribution by repeating the permutations of the data and after each permutation calculating $\Gamma_{\pi(Y) X}$. This generates a reference distribution of values for $\Gamma$ under the set of all $n$ ! permutations. Comparing the observed $\Gamma_{Y X}$ to this reference distribution provides a distribution-free way of testing the null hypothesis that $X$ and $Y$ are independent, under the model assumption that the distribution of the variables arranged in $X$ and $Y$ is permutationally invariant under permutation of the objects $O_{1}, \ldots, O_{n}$. In practice, it suffices to use a relatively small random sample $(1,000$ or 10,000$)$ from the set of all $n$ ! permutations to approximate this reference distribution (see also Pitman, 1937; Mantel, 1967; Jackson \& Somers, 1989).

Hence, the QAP provides a permutation- or randomization-based nonparametric test of dependence between two square matrix variables of the same size, which may represent, e.g., distances or similarities between objects, or a relation in a group of social actors. The QAP also directly provides the $p$-value calculated as the relative frequency of the values of the statistic, in the null distribution, that are larger than or equal to the observed value.

Validity of randomization tests hinges on the exchangeability of values of variables under permutation. In this context, the objects generating the rows and columns are assumed to be exchangeable. Of all the $(n(n-1))$ ! possible permutations of the matrix elements not considering the diagonal, only those $n$ ! permutations are used that respect the structure imposed by these objects, and this keeps intact the autocorrelation structure of the observations arranged in the square matrix.

\section{MRQAP Approaches}

In Multiple Regression-QAP (MRQAP), the study of the dependence between matrices $X$ and $Y$ is compounded because there are other variables $Z$ for which the association between $X$ and $Y$ must be controlled. Multiple regression is often used for hypothesis testing based on dyadic data in social sciences. For example, in their analysis of predicting the volume of international trade Krempel and Plümper (2003) use a distance measure, the gross domestic product of countries and dummy variables that indicate whether two countries share a border and have a coast on the same ocean. On a micro level, Krackhardt and Kilduff (1990) study the effect of friendship ties on cultural agreement among individuals and control for tenure differences and formal organizational position.

In this paper all variables are considered to be mean-centered over all observations excluding the diagonal values, which are ignored. Overviews and comparisons for vector data are given by Anderson and Legendre (1999) as well as Anderson and Robinson (2001), for analysis of variance (ANOVA) by Anderson and Ter Braak (2003), and for distance matrices by Legendre (2000). The approach taken is the multiple linear regression approach: it is assumed that $Y$ depends linearly on $Z$, and it is tested whether there is an additional (linear) dependence on $X$. It should be noted that in all cases, for vector and matrix data alike, linear dependence between variables is proposed as an approximation that is convenient and, hopefully, a good representation of reality. In the case of real-valued numerical data, the approximation is often quite good, and it may be expected that there is sufficient robustness for small deviations from linearity. For binary and other coarsely grained discrete data, however, linear models are less appropriate and 
other models for spatial or network data, such as those proposed by Snijders et al. (2006) or Heagerty and Lele (1998), may be used.

The basic linear model for square matrix data considered here is

$$
Y=\beta X+Z \gamma+E
$$

where $Y, X$, and $E$ are $n \times n$ matrices, $\beta$ is a scalar, $Z$ is an $n \times n \times q$ array, and $\gamma$ is $q \times 1$. The diagonals of the matrices are always ignored. The null hypothesis is

$$
H_{0}: \beta=0 \text {. }
$$

The variables $Z$ and $X$ are not assumed to be independent. Specifically, we assume between these variables the linear model

$$
X=\delta Z+V,
$$

where $V$ is an $n \times n$ matrix. The situation $\delta \neq 0$ will be called collinearity. The nonparametric approach to square matrix data means here that the residuals associated with the $n$ objects are exchangeable or, equivalently, the matrices $E$ and $V$ are invariant under permutations of rows and columns simultaneously by the same permutation. Whenever the term permutation is used, it will be assumed that this permutation acts on rows and columns simultaneously and in the same way, as described above for the QAP.

It will be convenient to use the following notation. The OLS estimator of $\beta$-the partial regression coefficient - for the vectorized data is denoted by

$$
\hat{\beta}=\hat{\beta}(Y, X \mid Z) \text {. }
$$

We use the notation $\pi(Y)$ for the data matrix $Y$ where both rows and columns are rearranged by the permutation $\pi$.

As stated earlier, the permutation principle can be applied to various test statistics. The QAP was explained above using as test statistic an association coefficient such as the elementwise correlation coefficient between the two matrices $X$ and $Y$. Consider first the bivariate case where the third variable $Z$ is absent from the model. Then the dependence between $X$ and $Y$ can be tested by the correlation coefficient $r$ in a permutation distribution. The following statistics can be used:

1. the correlation coefficient $r=r(X, Y)$

2. the $t$ transform $t=\sqrt{n(n-1)-2} r / \sqrt{1-r^{2}}$, which has an exact $t$ null distribution only if $E$ is normally distributed and there is no network autocorrelation, i.e., all elements of the matrices $E$ are independent

3. or the estimated regression coefficient

$$
\hat{\beta}=\frac{s_{Y}}{s_{X}} r
$$

where the standard deviations refer to the vectorized data

In the bivariate QAP permutation test the estimated regression coefficient is a constant multiple of the correlation coefficient since the two standard deviations remain constant under the permutations. Therefore, these three test statistics will lead to the same results for the bivariate QAP. For the MRQAP approaches, where more complicated statistics must be used to control for the dependence on the third variable $Z$, the equivalence between these three possibilities no longer holds. Consequently, in the MRQAP case, we cannot assume that these three apparently equivalent measures of association will behave the same way under random permutations. Indeed, as we will show, they do not. 
Different methods of data permutation have been proposed. They can be classified as "raw data" permutation methods and "residual" methods, as will be explained below. Some of these methods, when applied to some statistics may not have desirable properties. For example, Kennedy and Cade (1996) show that the method of permuting the raw $Y$-matrix yields substantial biased results with the use of a nonpivotal statistic. A statistic is defined to be pivotal when the distribution of the statistic under the null hypothesis is independent of the "nuisance" parameters; in our case, the nuisance parameters may be taken to be the residual variance and the regression coefficient $\delta$ in (3). When considering the null hypothesis $\beta=0$ under permutational invariance (rather than independence) of the residuals, the nuisance parameters also include the type of dependence (autocorrelation) of the residuals $E$. The partial regression coefficient is not pivotal. Thus, the Kennedy and Cade (1996) result suggests that it would be inappropriate to use the regression coefficient as a test statistic under the permutation of the raw $Y$-matrix. Furthermore, Anderson and Robinson (2001) show that although most "residual" methods asymptotically give the same results, they differ in the ability to approximate an exact test in small samples. It can be concluded from this literature that in some procedures it is clearly better to use a pivotal statistic; in other cases, there might be no important difference. We will explore the sensitivity of each of the MRQAP methods by using it with both pivotal statistics and nonpivotal statistics in our test below.

\subsection{Raw Data Methods: $X$ and $Y$ Permutations}

Intuitively attractive approaches to MRQAP for the three matrix case as given in (1), are two simple extensions of the bivariate case: the $X$-permutation and $Y$-permutation procedures (e.g., Oja, 1987; Smouse, Long, \& Sokal, 1986). In the former, we permute the $X$ matrix associated to the tested multiple-regression coefficient and subsequently estimate the multiple regression coefficients for the permuted data sets to generate its reference distribution. In the latter we permute $Y$.

The $H_{0}$ for the permutation of $X$ is that $Y$ is not related to $X$ although it could be related to $Z$. Now, permuting $X$ destroys the relationship between $X$ and $Z$ and thereby violates the ancillarity principle (Welch, 1990; Ter Braak, 1992; Anderson \& Legendre, 1999), which states that the dependence between these independent variables should be kept intact in the permutational procedure. It could be expected that this lack of respect for the dependence between $X$ and $Z$ will lead to incorrect type I error rates in cases of collinearity.

The $H_{0}$ for the permutation of $Y$ is that $Y$ is not related to $X$ and $Z$ taken together. It hence is a test for the hypothesis that both $\beta$ and $\gamma$ are 0 , not a test of $\beta$ alone (controlling for a possibly nonnull effect of $Z$ ). However, various researchers have been using the test for the latter purpose. This may explain that the literature on permutation tests has over the years presented contradictory advice on the use of the $Y$-permutation approach to MRQAP. Although Smouse et al. (1986) suggest this method is not the best approach, it has been applied in many studies. In social network analysis it has been applied perhaps unwittingly by many researchers, because it was the only approach to MRQAP implemented in the main network analysis software package UCINET, versions 3 to 6.1 (Borgatti, Everett, \& Freeman, 2002).

Manly (1997) argued for the $Y$-permutation, but this approach was criticized by Kennedy and Cade (1996), especially if a nonpivotal statistic is used. Anderson and Robinson (2001) found that the asymptotic significance level of the $Y$-permutation test is indeed equal to the nominal level when an asymptotically pivotal statistic is used. An asymptotically pivotal statistic does not depend on any unknown parameters and thereby adjusts for any nuisance parameters that are not of interest to the test (Anderson \& Robinson, 2001). The partial correlation coefficient and the corresponding $t$-statistics are examples of asymptotically pivotal statistics, independent of firstand second-moment population parameters. They are monotonic to each other, and can therefore be used equivalently for a significance test of individual multiple regression coefficients. 
The following shows the effects of collinearity (a linear relation between $X$ and $Z$ corresponding to $\delta \neq 0$ in (3)) when one uses the partial regression coefficient as reference statistic. The following relation holds between the partial regression coefficient $b_{Y X . Z}$ and the partial correlation coefficient $r_{Y X . Z}$ :

$$
b_{Y X . Z}=r_{Y X . Z} \frac{\sqrt{1-r_{Y Z}^{2}}}{\sqrt{1-r_{Z X}^{2}}} \frac{s_{Y}}{s_{X}} .
$$

Under $Y$-permutation the variable $Y$ is replaced with $\pi(Y)$. The partial regression coefficient under permutation is

$$
b_{\pi(Y) X . Z}=r_{\pi(Y) X . Z} \frac{\sqrt{1-r_{\pi(Y) Z}^{2}}}{\sqrt{1-r_{Z X}^{2}}} \frac{s_{Y}}{s_{X}} .
$$

Under random permutations, $E\left(r_{\pi(Y) Z}\right)$ will be approximately 0 , but this is not necessarily true for the nonpermuted data. Now, if $\rho_{Y Z} \neq 0$, the values $r_{\pi(Y) Z}$ under random permutations will tend to be lower and, consequently, the reference distribution consisting of the values of (7) will tend to be larger in absolute value. This will lead to a conservative test with an unnecessarily low power.

Similarly, when we use the multiple regression coefficient under $X$-permutation, the reference values are

$$
b_{Y \pi(X) . Z}=r_{Y \pi(X) . Z} \frac{\sqrt{1-r_{Y Z}^{2}}}{\sqrt{1-r_{Z \pi(X)}^{2}}} \frac{s_{Y}}{s_{X}} .
$$

Under permutation, $E\left(r_{Z \pi(X)}\right)$ is approximately 0 , which again is not necessarily true under the null hypothesis $\left(\beta_{Y X . Z}=0\right)$ ). If $\rho_{Z X} \neq 0$ the reference values will tend to be too small in absolute value, leading to a liberal test which is undesirable.

It was proved by Anderson and Robinson (2001) that for permutation tests on i.i.d. data, the asymptotic permutational distribution of the partial correlation coefficient, which is the correlation between the residuals of $Y$ and $X$, under permutations of $Y$, is standard normal under the null hypothesis. This implies that the $Y$-permutation approach gives asymptotically a test with the correct type I error rate when a pivotal statistic is used.

\subsection{Residual Permutation Methods}

Next to the $X$-permutation and $Y$-permutation approaches, various approaches have been proposed in the literature that are based on fitting a regression model, calculating the residuals, and permuting these residuals. These residuals are estimates of $E$. If the values of $E$ would be known exactly, then an exact test could be based on these, but the fact that only approximations to $E$ are available leads to a diversity of solutions, none of which are exact (Anderson \& Robinson, 2001).

3.2.1. The Freedman-Lane Approach to Permutations. First, we follow the procedure proposed for the usual linear model by Freedman and Lane (1983) and discussed further by various other authors (see Anderson \& Robinson, 2001). In our case the structure is more complicated because we are dealing with square arrays expressing relational data, but the basic approach can be similar. 
Now consider the general case that we do have the third variable $Z$ in the model. The null hypothesis is that there is no effect of $X$ on $Y$; all variation in $Y$ can be explained by $Z$, or is modeled by $E$. The proposal by Freedman and Lane (1983) is, first, to estimate the effect of $Z$ alone on $Y$, yielding the residuals

$$
\hat{\epsilon}_{Y Z}=Y-\hat{\gamma} Z
$$

where $\hat{\gamma}$ is the OLS regression parameter estimate for the reduced model

$$
Y=\gamma Z+E
$$

The residuals are equal to

$$
\hat{\epsilon}_{Y Z}=(\gamma-\hat{\gamma}) Z+E,
$$

which, under $H_{0}$, will be close to $E$ if $n$ is large and the model is well specified. Second, they propose to permute the residuals and calculate a new hypothetical $Y$-value made of the fitted portion of the regression equation plus the permuted residuals:

$$
Y^{\pi}=\hat{\gamma} Z+\pi\left(\hat{\epsilon}_{Y Z}\right)
$$

which is different from $\pi(Y)$ introduced earlier. For each of the $n$ ! permutations $\pi$, the pivotal $F$-statistic is calculated where $Y^{\pi}$ is regressed on $X$ controlling for $Z$, i.e., the model

$$
Y^{\pi}=\beta X+\gamma Z+E
$$

is applied. This statistic may be called $F\left(Y^{\pi}, X \mid Z\right)$. For a one-dimensional $X$-variable, this is a constant multiple of the squared $t$-statistic. The result proved by Freedman and Lane in the usual linear model is that for large $n$ and normal error, the permutation distribution of this statistic is approximately a multiple of chi-squared, and the same as the large sample distribution of the usual parametric $F$ test. For permutation testing, the important thing is that this asymptotic distribution does not depend on the unknown $\delta$ or $\gamma$, and that under $H_{0}: \beta=0$, it is the same as the asymptotic distribution that would be obtained if the true $E$ would be used instead of $\hat{\epsilon}_{Y Z}$. This implies that the permutation test of the null hypothesis, defined by $(1,2)$, and the assumption of permutational invariance over $E$, has approximately for large $n$ the correct level of significance. This result is subject to some assumptions which say basically that there are no outliers in the $Z$ or $Y$ variables.

If $Z$ is absent from the model, then the two procedures presented in the preceding subsection are also equivalent to this procedure, because permuting $X$ with constant $Y$ or permuting $Y$ with constant $X$ is equivalent if there is no $Z$ variable.

Comparing the $F$ test on the residuals with formula (13), and using (12) and (11), we have

$$
\begin{aligned}
\hat{\beta}\left(Y^{\pi}, X \mid Z\right) & =\hat{\beta}\left(\hat{\gamma} Z+\pi\left(\hat{\epsilon}_{Y Z}\right), X \mid Z\right) \\
& =\hat{\beta}\left(\pi\left(\hat{\epsilon}_{Y Z}\right), X \mid Z\right) \\
& =\hat{\beta}(\pi((\gamma-\hat{\gamma}) Z), X \mid Z)+\hat{\beta}(\pi(E), X \mid Z),
\end{aligned}
$$

and, in the last line, the second term has a permutation invariant distribution while the first term is negligible when $n$ is large and therefore $(\gamma-\hat{\gamma})$ is small. This suggests that the Freedman-Lane approach of permuting residuals can be used not only for asymptotically pivotal statistics but also when using the partial regression coefficient as a test statistic. The same procedure for pivotal statistics was proposed by Anderson and Legendre (1999) for vector data and by Legendre (2000) for matrix data. 
This method, which we call the "Freedman-Lane Semi-Partialing" (FLSP) approach, might also be termed $Y$-conditional-semi-partialing regression approach, since the effect of $Z$ is partialed out from $Y$ and then the resulting $Y$ residuals are permuted and regressed on $X$ and $Z$. Note that in the process $Z$ is entered in the regression twice. The effect of $Z$ need not also be partialed out from $X$, since $Z$ is used in the regression anyway and $X$ and $Z$ are not permuted with respect to each other. Furthermore, putting in $\hat{\gamma} Z$ as is done in (12) does not change the $F$ statistic because $Z$ is being controlled for in model (13). Following Anderson and Robinson (2001) we could also use the partial correlation $r\left(\hat{\epsilon}_{Y Z}, X \mid Z\right)$ as a test statistic, and test it in the permutational distribution $r\left(\pi\left(\hat{\epsilon}_{Y Z}\right), X \mid Z\right)$. This will also be approximately the same as using the $F$ test as proposed by Freedman and Lane (see the "first idea" in their proof, which implies that asymptotically, $F\left(Y^{\pi}, X \mid Z\right)$ and $r^{2}\left(Y^{\pi}, X \mid Z\right)$ are functions of each other).

3.2.2. Double Semi-Partialing. We now introduce a new approximately exact test that follows a similar logic as the FLSP and hence complements the set of similar existing permutation tests. Whereas the FLSP approach conditions the test statistic under permutation on the relationship between $Y$ and $Z$, we could follow an analogous reasoning and condition it on the relationship between $X$ and $Z$. Although this is a logical analogue of the Freedman-Lane approach, it seems not to have been proposed before.

Similar to (9) we define

$$
\hat{\epsilon}_{X Z}=X-\hat{\delta} Z
$$

where $\hat{\delta}$ is the OLS estimate for the model

$$
X=\delta Z+V
$$

In the new method these residuals are permuted and the model

$$
Y=\beta \pi\left(\hat{\epsilon}_{X Z}\right)+\gamma Z+E
$$

is used to obtain reference values for the test statistic. The rationale here is that under the null hypothesis $\beta=0$, the reference model (17) for $Y$ is the same as the original model in (1), and if the estimation error $\hat{\delta}-\delta$ is negligible, the permutational invariance assumption for $V$ implies that

$$
\pi\left(\hat{\epsilon}_{X Z}\right)=\pi((\delta-\hat{\delta}) Z+V)
$$

has the same distribution as $V$.

This approach has some parallels to the Freedman-Lane approach that focuses on generating a permuted $Y$. More, specifically $Y^{\pi}$, which as is shown in (14), is equal to using $\pi\left(\hat{\epsilon}_{Y Z}\right)$ (see also Anderson \& Legendre, 1999). Instead of generating $Y^{\pi}$ this procedure focuses on creating an $X^{\pi}$, or rather, similar to what Anderson and Legendre (1999) propose to simplify things, focuses on $\pi\left(\hat{\epsilon}_{X Z}\right)$.

We refer to this new approach as Double-Semi-Partialing (DSP) regression since the effect of $Z$ is partialed out of $X$ and then the resulting residuals $\hat{\epsilon}_{X Z}$ are permuted and entered in a regression of $Y$ on both $\hat{\epsilon}_{X Z}$ and $Z$. As such $Z$ enters the regression twice, hence the "double." Where the FLSP approach minimizes the correlation between the ancillary variables and the dependent variable under permutation, the DSP approach minimizes the correlation between the focal variable and the control variables under permutation. Both methods therefore respect the ancillarity principle as they condition on the nuisance statistics. Simulations below will have to determine which of these two methods minimizes the effect of the "nuisance" parameters under permutation. 
3.2.3. Partialing. Many other approaches could be considered with respect to what is partialed out and what is permuted. One of the possibilities is to estimate residuals $E$ under the full model in (1) rather than the reduced model in (10), as proposed by Ter Braak (1992). However, Anderson and Robinson (2001) obtained for this method power properties that were inferior to some other methods, which was confirmed by some of our own simulations (not further reported below). Therefore, this approach is not further elaborated here.

Another approach proposed in the MRQAP literature is called partialing (Smouse, Long, \& Sokal, 1986; Krackhardt, 1988; Kennedy, 1995). This approach tests $r\left(\hat{\epsilon}_{Y Z}, \hat{\epsilon}_{X Z}\right)$ in the distribution of reference values $r\left(\hat{\epsilon}_{Y Z}, \pi\left(\hat{\epsilon}_{X Z}\right)\right)$. Anderson and Legendre (1999) found the partialing approach to be incorrect for the case of multiple regression, and Legendre (2000) found the partial approach to be incorrect for the partial Mantel test.

Anderson and Legendre (1999) review many different permutation proposals for the usual linear model (with vector data). These authors conclude that the Freedman-Lane proposal (here called FLSP) applied to the partial correlation coefficient corresponds best with the test based on the unobserved true residuals $E$, i.e., results coming closest to the actual significance level as well as giving the highest power. For the partialing approach, which they call the Kennedy approach in reference to Kennedy (1995), they show that this test will always result in a higher level of type I errors than the Freedman-Lane approach.

In the remainder of this paper we analyze how different MRQAP tests on partial regression coefficients perform in terms of accuracy and power when we impose different collinearity and autocorrelation conditions. Also, we compare the consequences of using nonpivotal statistics in "raw data" permutation methods. This extends the simulation study of MRQAP tests for matrix data carried out by Legendre (2000), who considered distributions representing distance matrices, whereas we specifically focus on dependence structures that are typical for network data.

\section{Method}

This section presents a simulation study using model specifications consistent with the type of network data to which MRQAP approaches have been applied in the literature (e.g., Nelson, 1989; Krackhardt \& Kilduff, 1999; Borgatti \& Cross, 2003). Autocorrelations in the sense of within-row and within-column correlations range from nil to strong. Normally distributed data are considered as well as skewed and discrete data.

For getting simulated matrices with correlations within rows, as well as within columns, and with different kinds of nonnormal data, it is convenient to use the formula

$$
M_{i j}=R_{i}+C_{j}+E_{i j}
$$

where the row contributions $R_{i}$, the column contributions $C_{j}$, and the cell contributions $E_{i j}$ are independent $(M=X, Y, Z)$. When the row and column contributions have the same variance $\operatorname{var}\left(R_{i}\right)=\operatorname{var}\left(C_{j}\right)=\sigma_{1}^{2}$ and the cell contributions have variance $\operatorname{var}\left(E_{i j}\right)=\sigma_{2}^{2}$, then the withinrow as well as the within-column autocorrelation is $\rho_{a}=\sigma_{1}^{2} /\left(2 \sigma_{1}^{2}+\sigma_{2}^{2}\right)$.

In the simulation, matrices are of dimensions $10 \times 10$. Hence, since diagonal values are ignored, simulations are based on 90 dyad level observations. The following other choices are made for the simulations:

1. four levels of autocorrelation, $\rho_{a}=.00, .15, .30, .45$

2. Normal distributions, Gamma distributions (skewed), Negative Binomial distributions (skewed and discrete)

3. three levels of skewness $(g)$, respectively $(.25, .75, .95)$ 
The reason for studying the effect of skewness of the simulated data on the performances of the tests is that in fact network data is often skewed.

We used results based on cumulants to derive the skewness of the sum of $R_{i}, C_{j}, E_{i j}$ in (19). Similar to moments, cumulants are descriptive parameters of distributions (Stuart \& Ord, 2005, p. 86). The skewness of the sum of independent random variables can be derived relatively easily, because the third cumulant equals the third central moment and the cumulant of a sum of independent random variables is the sum of the cumulants of those variables (Stuart \& Ord, 2005, pp. $89,156)$. Hence, the sum of the third central moments of independent random variables equals the third central moment of their sum.

Denoting the skewness of some random variable by $\lambda$, the third central moment by $\mu_{3}$, and the standard deviation by $\sigma$, skewness is defined by $\lambda=\mu_{3} / \sigma^{3}$. The independence of $R_{i}, C_{j}$, and $E_{i j}$ implies

$$
\mu_{3}\left(R_{i}+C_{j}+E_{i j}\right)=\mu_{3}\left(R_{i}\right)+\mu_{3}\left(C_{j}\right)+\mu_{3}\left(E_{i j}\right)
$$

and

$$
\sigma^{2}\left(R_{i}+C_{j}+E_{i j}\right)=\sigma^{2}\left(R_{i}\right)+\sigma^{2}\left(C_{j}\right)+\sigma^{2}\left(E_{i j}\right)
$$

which yields

$$
\lambda\left(R_{i}+C_{j}+E_{i j}\right)=\frac{\lambda\left(R_{i}\right) \sigma^{3}\left(R_{i}\right)+\lambda\left(C_{j}\right) \sigma^{3}\left(C_{j}\right)+\lambda\left(E_{i j}\right) \sigma^{3}\left(E_{i j}\right)}{\left\{\sigma^{2}\left(R_{i}\right)+\sigma^{2}\left(C_{j}\right)+\sigma^{2}\left(E_{i j}\right)\right\}^{3 / 2}} .
$$

Given a desired level of skewness for $M_{i j}$, and given the variances chosen earlier to control the levels of autocorrelation in the data, this now allows us to choose the levels of skewness for $R_{i}$, $C_{j}$, and $E_{i j}$.

Also, we impose different levels of correlation between $Y$ and $X$ implied by the joint dependence ("collinearity") of $X$ and $Y$ on $Z$. More specifically, in this case,

$$
r_{Y X}=r_{Y Z} r_{Z X}
$$

We enforce that $r_{Y Z}=r_{Z X}$, hence $\sqrt{r_{Y X}}=r_{Y Z}=r_{Z X}$. The value of $r_{Y X}$ is a spurious correlation between $Y$ and $X$, reflecting an apparent relationship between $Y$ and $X$ that is in fact induced by the third variable $Z$. When we control for $Z$ in a multiple regression setting the bivariate effect of $X$ on $Y$ disappears. Henceforth, "spuriousness" hence indicates the correlation between $Y$ and $X$ due to the presence of $Z$.

To represent the collinearity conditions we use a $3 \times 3$ correlation matrix $T$ that represents the correlations between $Y, X$, and $Z$. Furthermore, we vectorize and concatenate our square data matrices into the data matrix $D$ of dimension $n(n-1) \times 3$. A Cholesky decomposition of $T$ provides the weights for the construction of the correlated data set $D T$. From this correlated data set $D T$ of dimension $(n(n-1) \times 3)$ we reconstruct the 3 square $(n \times n)$ variable matrices $Y$, $X$, and $Z$. In our simulations the collinearity conditions expressed by $\rho_{X Z}$ increase by .3 from 0 to .9. In the case of integer values in the negative binomial count data sets, we round the values of $Y, X$, and $Z$ to the nearest integer.

In the "type I error" simulations we record the percentage of rejections (based on 1000 runs per step) of the (true) null hypotheses, where there is no relation between $Y$ and $X$ (occurrences of type I error). We assess robustness of MRQAP tests by evaluating results at a significance level of $\alpha=.10$ (similar to Krackhardt, 1988). To asses the consistency of the different tests based on the Monte Carlo simulations we use the lower and upper boundaries of the $99 \%$ expected outcome interval (Edgington, 1995). 
For the power study we generated data for which the null hypothesis was false, i.e., $\beta \neq 0$. Data were generated in a similar fashion as in the "type I error" study described above. The difference was that we set the correlation as

$$
r_{Y X}=r_{Y Z} r_{Z X}+.1
$$

where again $r_{Y Z}=r_{Z X}$.

In the power study we thus vary the level of spuriousness given an extra correlation of .1 between $Y$ and $X$, and we vary the levels of autocorrelation in the data.

\section{Results}

The consequences for type I error of using the nonpivotal statistic $\hat{\beta}$ in the "raw data" methods are shown in Figures 1(a) and 1(b). They show how increasing spuriousness due to collinearity of $Y$ and $X$ with a third variable $Z$ harms the accuracy of these MRQAP tests. The MRQAP test using $\hat{\beta}$ with the $Y$-permutation will almost never reject the null hypothesis when spuriousness increases (see Figure 1(a)). This should translate in reduced power when there is an effect. In contrast, the MRQAP test that uses $\hat{\beta}$ with the $X$-permutation almost always rejects the null hypothesis (see Figure 1(b)). Both tests are therefore uninformative. Figures 2(a) and 2(b) show that under these conditions pivotal statistics do give results close to the expected $\alpha$ level of .10 for the same set of permutations on the same data. These results corroborate the conclusions of Legendre (2000) for matrix and those of Legendre and Anderson (1999) and Anderson and Robinson (2001) for i.i.d. (vector) data.

Note that in Figures 1 and 2 the results seem to be independent of the level of autocorrelation. A close inspection of our results for the other methods (partialing, FLSP, DSP) shows similar results (see Figures 1(c) to 1(e) and 2(c) to 2(e)). This confirms and extends the results of Krackhardt (1988) as it shows that statistical bias of MRQAP tests is minimal under varying conditions of autocorrelation and spuriousness-but only when a pivotal statistic, such as $t$, is used.

However, the methods do differ in their ability to approximate the exact test. The $99 \%$ expected outcome interval for 1000 runs and $\alpha=.10$ is $\langle .075-.125\rangle$ (Edgington, 1995). If rejection proportions fall outside this interval they significantly differ from the nominal value $\alpha=.10$ and this indicates a biased reference distribution.

The results for Normal data (Figure 2) show that all values remain within the expected outcome interval. It is remarkable that this fact is also true for the "raw data" methods. Despite the good performance of "raw data" methods, overall, these methods typically give less robust results compared to the semi-partialing methods for a nonpivotal statistic (see Figure 1). Therefore, in the sequel we will not focus on our results on the "raw data" methods. Partialing gives type I error rates that are always larger than or equal to the results of FLSP (all data points in Figure 2(c) are higher than comparable points in Figure 2(e)). This is consistent with the findings of Anderson and Robinson (2001). Furthermore, the results show that DSP is often closer to the expected type I error level than partialing (for Normal data the average of absolute deviation from .10-level over 16 simulated autocorrelation-collinearity conditions are .008 and .013 , respectively). Because FLSP has been analytically shown to be superior to partialing (Anderson \& Robinson, 2001) we subsequently focus our results on the differences between FLSP and DSP.

Simultaneously high levels of skewness and spuriousness harm the MRQAP tests. This can be seen from the increasing type I error rates in Figures 3 and 4 as both increase as skewness and spuriousness increases. Figure 3 shows that Gamma data with $g=.250$ do not generate 

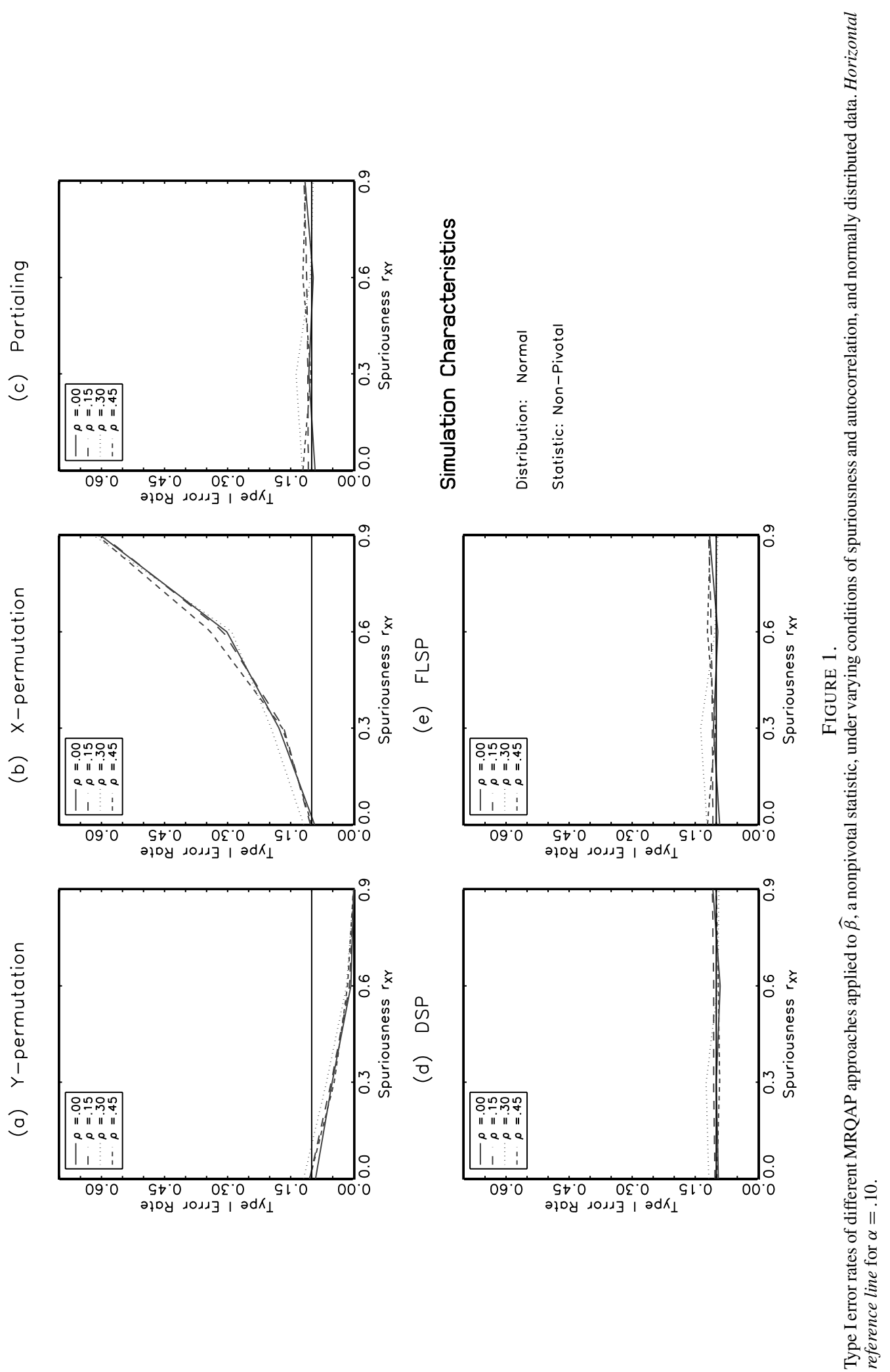

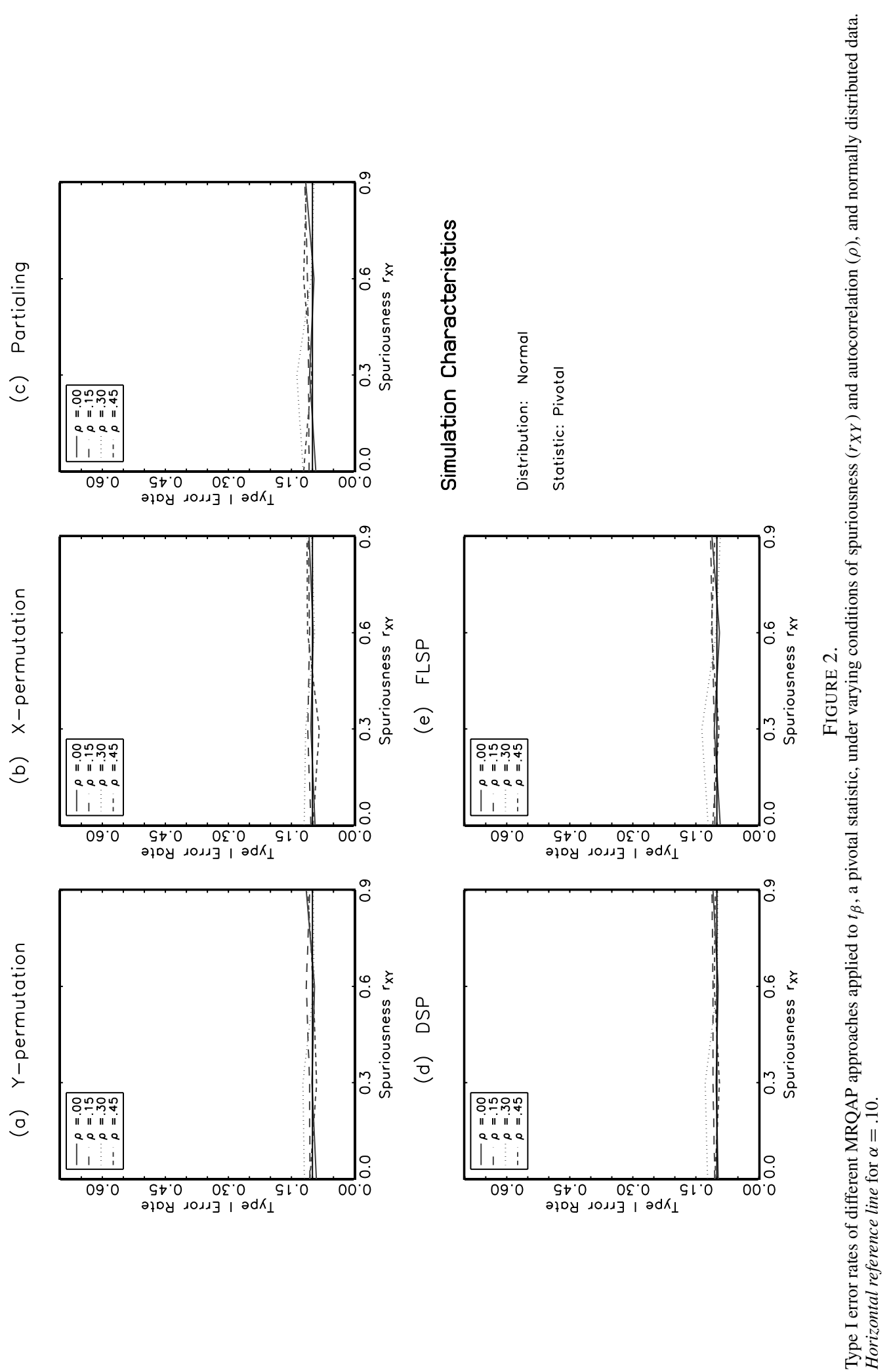
(a) DSP $(g=.25)$

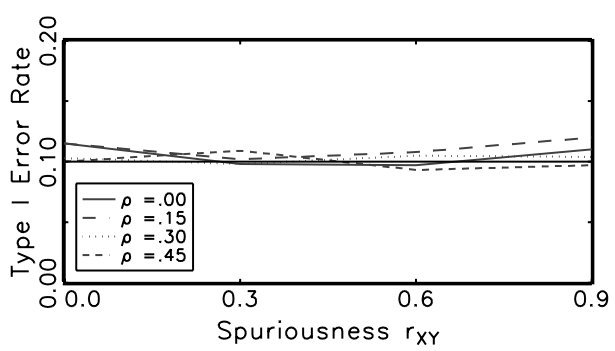

(c) DSP $(g=.75)$

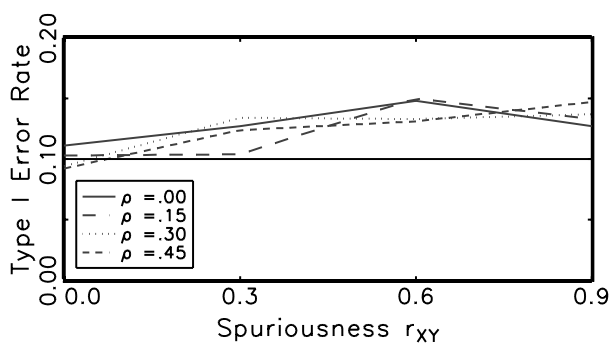

(e) DSP $(g=.95)$

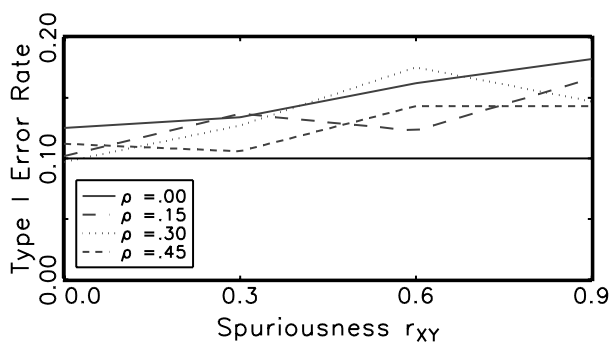

(b) FLSP $(g=.25)$

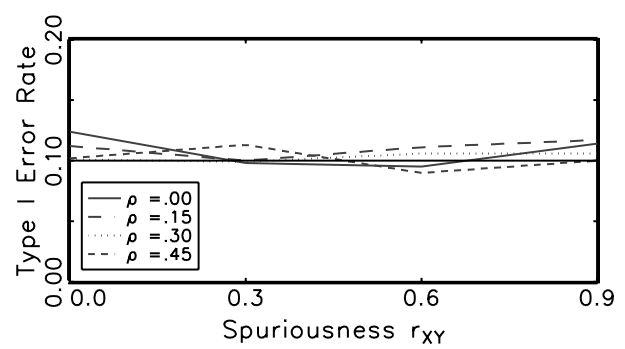

(d) FLSP $(g=.75)$

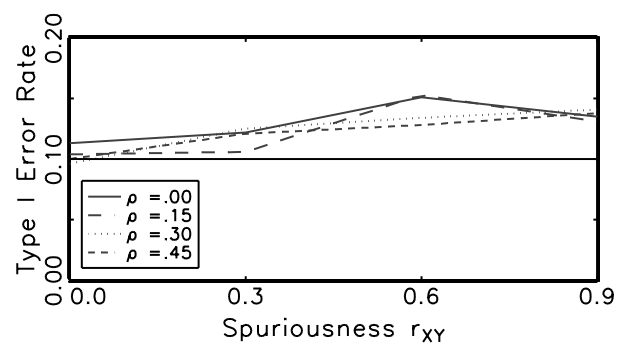

(f) FLSP $(g=.95)$

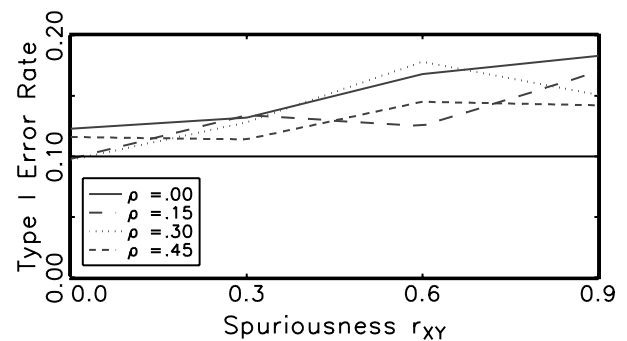

Figure 3.

Type I error rates of different MRQAP approaches applied to $t_{\beta}$, under varying conditions of spuriousness $\left(r_{X Y}\right)$, autocorrelation $(\rho)$, and skewness $(g)$, and gamma distributed data. Horizontal reference line for $\alpha=.10$.

conditions that cause a serious problem for FLSP and DSP. Indeed, these results are comparable to the results generated with normal data. However, for high levels of skewness ( $g=.75$ and $g=.95$ ) we see that rejection rates structurally cross the upper bound of .125 when spuriousness is also high $\left(r_{Y X}>.30\right)$. Overall, results for the Gamma distributions and Negative Binomial distributions are similar, although the results for the various MRQAP tests seem to be slightly better for Gamma-distributed data (compare Figures 3 and 4).

The results of the power study with normal data are summarized in Figure 5 (similar results were obtained for the skewed distributions). The results show that the power of the different methods depends heavily on both the levels of spuriousness and of autocorrelation in the data. Figure 5 shows that autocorrelation decreases power, while spuriousness enhances power given that formula (24) is used to define the point under the alternative hypothesis where power is studied. The fact that autocorrelation decreases power results from the fact that autocorrelated data contain less statistical information that can be utilized in this analysis. The results show that for both types of data, when autocorrelation becomes excessive, power of the test deteriorates heavily. 
(a) DSP $(g=.25)$

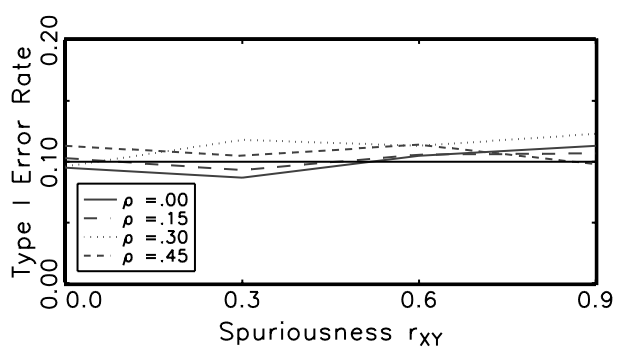

(c) DSP $(g=.75)$

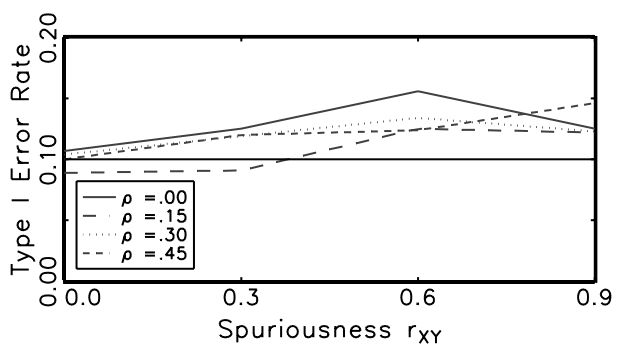

(e) DSP $(g=.95)$

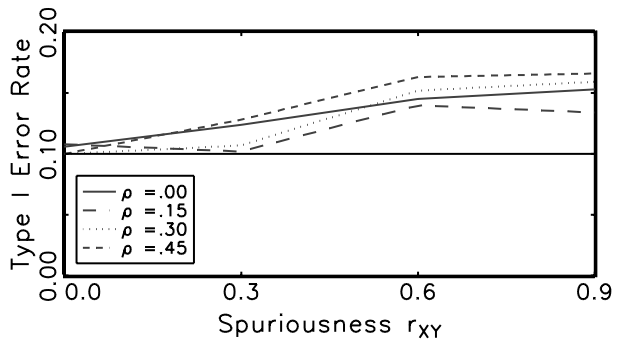

(b) FLSP $(g=.25)$

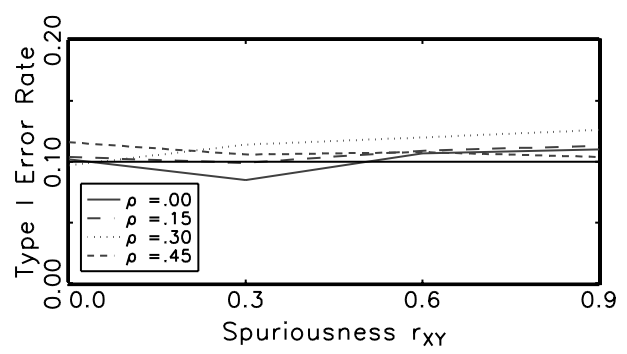

(d) FLSP $(g=.75)$

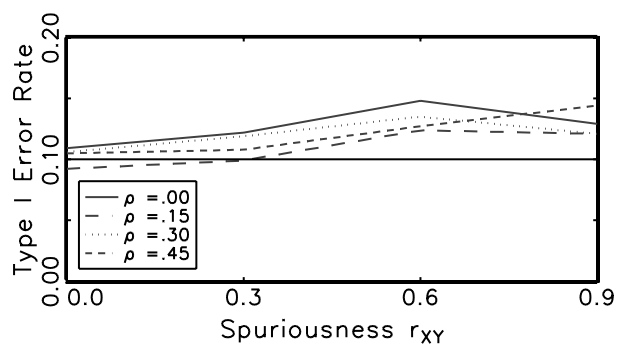

(f) FLSP $(g=.95)$

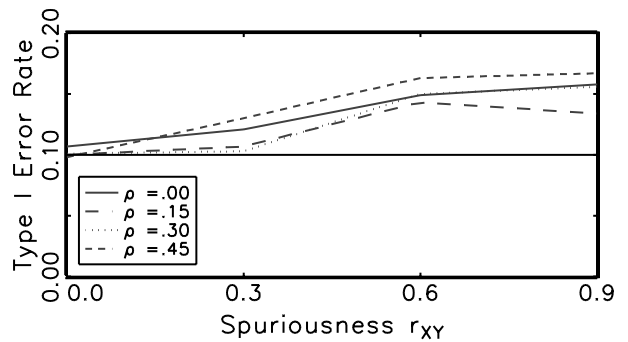

FIGURE 4.

Type I error rates of different MRQAP approaches applied to $t_{\beta}$, under varying conditions of spuriousness $\left(r_{X Y}\right)$, autocorrelation $(\rho)$, and skewness $(g)$, and negative binomial distributed data. Horizontal reference line for $\alpha=.10$.

As would be expected partialing has the highest apparent power, which is due to its inflated rate of type I error. Because the rate of type I error is substantially higher than the significance level, the resulting tests are invalid (see Edgington, 1995, p. 13); thus they will not be considered further as a viable option here. The other methods give similar results, although the "residual" methods perform somewhat better than the "raw data" methods, as we expected. The power of the tests is lower for the skewed data than for the normally distributed data.

\section{Discussion and Conclusion}

Many studies in many different fields employ MRQAP for analysis of network data in the linear model framework. It is a popular approach mainly because it both utilizes the benefits of multiple regression analysis and offers a solution to problems of autocorrelation that prohibit the use of standard statistical tests. However, the results obtained with MRQAP analyses are sensitive to particular methods and options implemented in standard software packages. In particular, the "raw data" permutation methods in this study that use nonpivotal statistics performed 

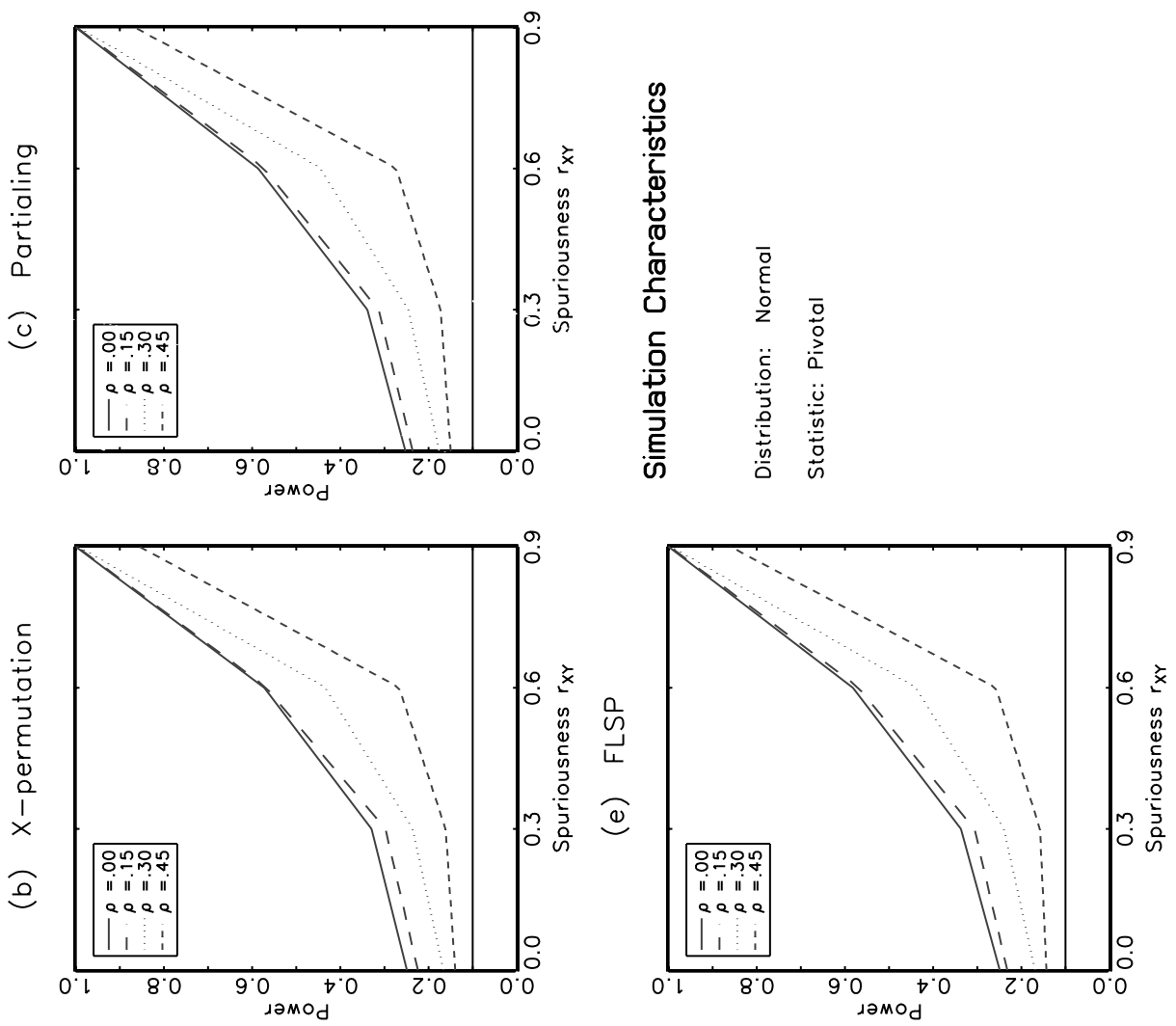

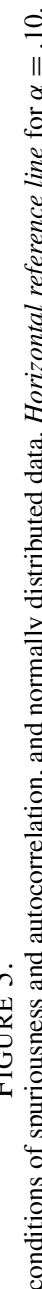

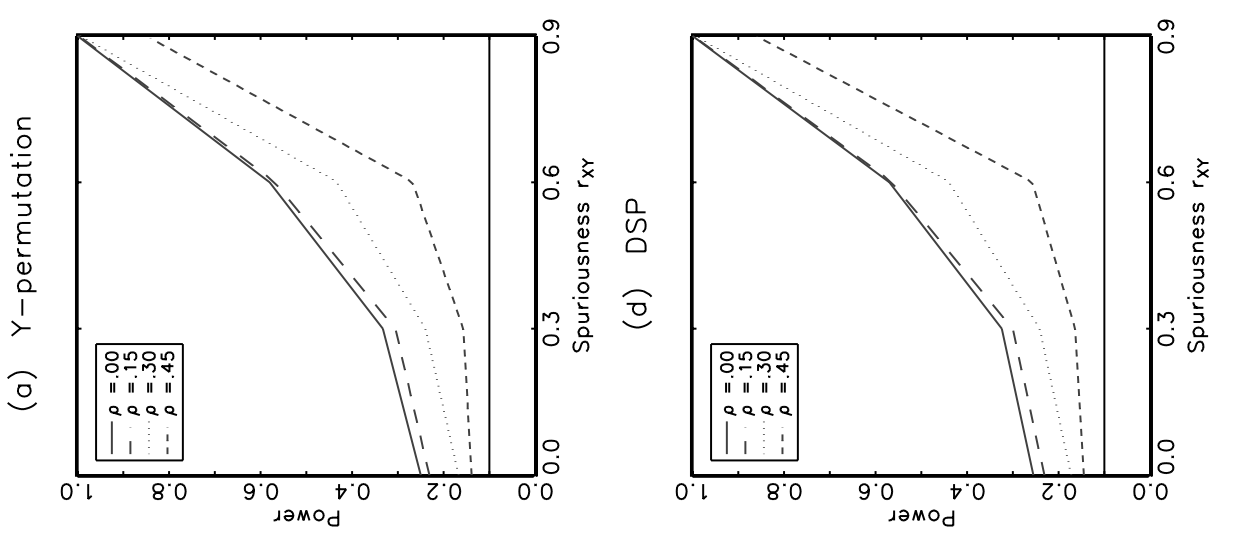

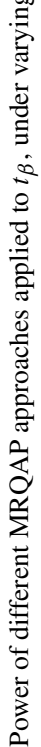


so poorly that they should be avoided, because rejection rates either rise far beyond acceptable levels or become extremely low. In the permutation test literature, these difficulties have been recognized (e.g., Anderson \& Robinson, 2001). Our analysis expands on this literature and adds to it more extensive insights into the problems of the different approaches in the case of structurally autocorrelated data such as encountered in network analysis. Also, it adds insights in how combinations of spuriousness and skewness affect the tests.

Despite the findings of Legendre (2000) that outliers inflate type I error for "raw data" methods (specifically, $Y$-permutation), some might insist that our results suggest that $Y$-permutation on a pivotal statistic is a good choice, especially for Gaussian data. This method would be the most efficient implementation option for software developers, because it simultaneously generates results for all coefficients.

However, simple reliance on a pivotal statistic presents its own problems, since it is not always possible to determine whether a statistic is pivotal in any given situation. A $t$-statistic and a partial correlation coefficient are pivotal statistics for multivariate normal distributions; but, when data stem from other distributions where higher moments are informative, these statistics are no longer guaranteed to be pivotal. Furthermore, the autocorrelation structures of network data imply that these statistics may no longer be pivotal under the null hypothesis of row and column permutational invariance considered in our paper.

The nonpivotal statistics in "raw data" methods may be statistically biased because of the effects of other independent variables. In the simulations presented here the "residual" methods do not suffer from these problems. As partialing is always inferior to FLSP, two recommendable methods remain, i.e., DSP and FLSP.

We must emphasize that this study is limited to conditions where the data conform to a general linear model, especially models where the dependent variable is continuous or at least countable integers (Poisson-like). In particular, we do not know the behavior of the different MRQAP approaches for models wherein the data are binary or ordinal, as is often found in social network data (see Snijders et al., 2006 for alternative models for these cases). It remains for further research to show what MRQAP approaches, possibly vastly different from those discussed here, have to offer for these types of models.

Taking all of these considerations into account, we suggest that in general the safest recommendation for researchers testing linear models of continuous or skewed network count data employ MRQAP analysis using a DSP or FLSP method with a $t$-statistic (or similarly judged pivotal statistic). When encountering other types of distributions or constraints and conditions than those studied here, we suggest following the methods we used to determine the best method for a particular problem.

\section{References}

Anderson, M.J., \& Legendre, P. (1999). An empirical comparison of permutation methods for tests of partial regression coefficients in a linear model. Journal of Statistical Computation and Simulation, 62(3), 271-303.

Anderson, M.J., \& Robinson, J. (2001). Permutation tests for linear models. Australian \& New Zealand Journal of Statistics, 43(1), 75-88.

Anderson, M.J., \& Ter Braak, C.J.F. (2003). Permutation tests for multi-factorial analysis of variance. Journal of Statistical Computation and Simulation, 73(2), 85-113.

Baker, F., \& Hubert, L. (1981). The analysis of social interaction data. Sociological Methods and Research, 9, 339-361.

Borgatti, S.P., \& Cross, R. (2003). A relational view of information seeking and learning in social networks. Management Science, 49(4), 432-445.

Borgatti, S.P., Everett, M.G., \& Freeman, L.C. (2002). Ucinet for windows: Software for social network analysis. Boston: Harvard Analytic Technologies.

Edgington, E. (1995). Randomization tests (3rd edn.). New York: Dekker.

Frank, O., \& Strauss, D. (1986). Markov graphs. Journal of the American Statistical Association, 81(395), 832-842.

Freedman, D., \& Lane, D. (1983). A nonstochastic interpretation of reported significance levels. Journal of Business and Economic Statistics, 1, 292-298.

Gibbons, D., \& Olk, P.M. (2003). Individual and structural origins of friendship and social position among professionals. Journal of Personality and Social Psychology, 84(2), 340-351. 
Heagerty, P.J., \& Lele, S.R. (1998). A composite likelihood approach to binary spatial data. Journal of the American Statistical Association, 93, 1099-1123.

Holland, P.W., \& Leinhardt, S. (1981). An exponential family of probability-distributions for directed-graphs. Journal of the American Statistical Association, 76(373), 33-50.

Hubert, L.J. (1987). Assignment methods in combinatorial data analysis (Vol. 73). New York: Dekker.

Jackson, D.A., \& Somers, K.M. (1989). Are probability estimates from the permutation model of Mantel's test stable? Canadian Journal of Zoology, 67, 766-769.

Kadushin, C. (1995). Friendships among the French financial elite. American Sociological Review, 60(2), $201-221$.

Kennedy, P.E. (1995). Randomization tests in econometrics. Journal of Business \& Economic Statistics, 13(1), 85-94.

Kennedy, P.E., \& Cade, B.S. (1996). Randomization tests for multiple regression. Communications in StatisticsSimulation and Computation, 25(4), 923-936.

Krackhardt, D. (1987). Qap partialling as a test of spuriousness. Social Networks, 9, 171-186.

Krackhardt, D. (1988). Predicting with networks-Nonparametric multiple-regression analysis of dyadic data. Social Networks, 10(4), 359-381.

Krackhardt, D., \& Kilduff, M. (1990). Friendship patterns and culture: The control of organizational diversity. American Anthropologist, 92, 142-154.

Krackhardt, D., \& Kilduff, M. (1999). Whether close or far: Perceptions of balance in friendship networks in organizations. Journal of Personality and Social Psychology, 76, 770-782.

Krempel, L., \& Plümper, T. (2003). Exploring the dynamics of international trade by combining the comparative advantages of multivariate statistics and network visualization. Journal of Social Structure, 4(1) (electronic).

Laumann, E.O., Marsden, P.V., \& Galaskiewicz, J. (1977). Community-elite influence structures: Extension of a network approach. American Journal of Sociology, 83(3), 594-631.

Laumann, E.O., \& Pappi, F.U. (1976). Networks of collective action: A perspective on community influence systems. New York: Academic Press.

Legendre, P. (2000). Comparison of permutation methods for the partial correlation and partial Mantel tests. Journal of Statistical Computation and Simulation, 67(1), 37-73.

Legendre, P., \& Anderson, M. (1999). Distance-based redundancy analysis: Testing multi-species responses in multifactorial ecological experiments. Ecological Monographs, 69, 1-24.

Legendre, P., Lapointe, F.-J., \& Casgrain, P. (1994) Modeling brain evolution from behavior: A permutational regression approach. Evolution, 48, 1487-1499.

Manly, B. (1997). Randomization, bootstrap, and Monte Carlo methods in biology (2nd edn.). London: Chapman \& Hall.

Mantel, N. (1967). The detection of disease clustering and a generalized regression approach. Cancer Research, 27(2), 209-220.

Mizruchi, M.S. (1990). Capitalist collective action: Competition, cooperation, and conflict in the coal industry, by J.R. Bowman. American Journal of Sociology, 96(2), 473-475.

Nelson, R.E. (1989). The strength of strong ties: Social networks and intergroup conflict in organizations. Academy of Management Journal, 32, 377-401.

Oden, N.L., \& Sokal, R.R. (1992). An investigation of 3-matrix permutation tests. Journal of Classification, 9(2), 275290.

Oja, H. (1987). On permutation tests in multiple regression and analysis of covariance problems. Australian Journal of Statistics, 29, 81-100.

Pattison, P.E. (1988). Network models: Some comments on papers in this special issue. Social Networks, 10(4), $383-411$.

Pitman, E. (1937). Significance tests which may be applied to samples from any population. Royal Statistical Society, Supplement, 4, 119-130.

Proctor, C.H. (1969). Analyzing pair data and point data on social relationships, attitudes and background characteristics of Costa Rican Census Bureau employees. Social Statistics Section, Proceedings of the American Statistical Association, pp. 457-465.

Smouse, P.E., Long, J.C., \& Sokal, R.R. (1986). Mutiple regression and correlation extensions of the Mantel test of matrix correspondence. Systematic Zoology, 35(4), 627-632.

Snijders, T., Pattison, P., Robins, G., \& Handcock, M. (2006). New specifications for exponential random graph models. Sociological Methodology, 36, 99-153.

Sorenson, O., \& Stuart, T.E. (2001). Syndication networks and the spatial distribution of venture capital investments. American Journal of Sociology, 106(6), 1546-1588.

Stuart, A., \& Ord, J.K. (2005). Kendall's advanced theory of statistics: Vol. 1. Distribution theory. London: Arnold.

Ter Braak, C. (1992). Permutation versus bootstrap significance tests in multiple regression and ANOVA. In K.-H. Jöckel, G. Rothe, \& W. Sendler (Eds.) Bootstrapping and related techniques. Berlin: Springer.

Wasserman, S., \& Pattison, P. (1996). Logit models and logistic regressions for social networks. 1. An introduction to Markov graphs and $p^{*}$. Psychometrika, 61(3), 401-425.

Welch, W. (1990). Construction of permutation tests. Journal of the American Statistical Association, 85, $693-698$. 